\title{
The CD79 $\alpha$ (HM47/A9) antibody is effective in distinguishing between primary hepatocellular carcinoma and primary intrahepatic cholangiocarcinoma
}

\author{
$\mathrm{HAO} \mathrm{LI}^{*}$ and CONGYANG LI* \\ Department of Pathology, People's Liberation Army 152 Hospital, Pingdingshan, Henan 467000, P.R. China
}

Received October 25, 2012; Accepted January 16, 2013

DOI: $10.3892 / \mathrm{ol} .2013 .1163$

\begin{abstract}
Hepatocellular carcinoma (HCC) and intrahepatic cholangiocarcinoma (ICC) are two major forms of primary liver cancer. The aim of this study was to investigate CD79 $\alpha$ (HM47/A9) antibody expression patterns in normal liver, HCC and ICC samples. HM47/A9 expression was examined in tissues surrounding liver cancer in adults, 8-week embryos and 20-week embryos. In total, 82 cases of HCC, 31 cases of ICC and 11 cases of combined HCC and cholangiocarcinoma (cHCC-CC) were reviewed. HM47/A9 expression was observed as early as 8-week embryo liver tissue and exhibited focal granular cytoplasmic positivity, which was maintained throughout life. All 82 HCC cases demonstrated cytoplasmic granular positivity for HM47/A9, while no ICC cases were immunostained with HM47/A9. No CC components in cHCC-CC expressed the HM47/A9 antibody. A high number of HCC components in $\mathrm{CHCC}-\mathrm{CC}$ showed positive staining for HM47/A9 [10/11 (90.9\%)]. Our results suggest that HM47/A9 may be employed effectively to differentiate HCC from ICC.
\end{abstract}

\section{Introduction}

Hepatocellular carcinoma (HCC) and intrahepatic cholangiocarcinoma (ICC) constitute two major forms of primary liver cancer. The two malignancies have different clinical and pathological features and prognosis, but in some cases histopathological overlap exists. Immunochemistry is thus required to facilitate differential diagnosis between HCC and ICC. To date, several antibodies, such as cytokeratin 19 (CK19), CD10, Hep Par 1, AFP, CA19-9, MOC31, glypican-3 and CEA, have

Correspondence to: Dr Hao Li or Dr Congyang Li, Department of Pathology, People's Liberation Army 152 Hospital, Room 42, Jianshe Road, Pingdingshan, Henan 467000, P.R. China

E-mail: lihao152154@yahoo.cn

E-mail: licongyang1977@yahoo.com.cn

${ }^{*}$ Contributed equally

Key words: primary hepatocellular carcinoma, primary intrahepatic cholangiocarcinoma, CD79 $\alpha$, HM47/A9 been used to differentiate between the two malignant tumor types (1-3).

CD79 $\alpha$ and CD79 $\beta$ belong to the Ig gene superfamily and contain one extracellular Ig-like domain, a transmembrane $\alpha$-helical region and a cytoplasmic domain (3). These glycoproteins form a disulfide-linked heterodimer in the B-cell receptor (BCR) and pre-BCR complexex (4). The CD79 $\alpha / \beta$ complex is critical to B-cell development, mediating signal transduction and promoting endocytosis of bound antigens for intracellular degradation and presentation to helper T cells (4). CD79 $\alpha$ protein is present in B-cell follicles in lymph nodes, plasma cells and the majority of circulating $B$ cells $(5,6)$, but absent in brain, colon, kidney, liver, muscle, pancreas and placenta tissues. CD79 $\alpha$ expression is not limited to B cells, as it is also detected in the normal early myeloid precursors and megakaryocytes (7). We (unpublished data) disclosed strong immunoreactivity of HM47/A9 with hepatocytes, but not with the bile canaliculus or interlobular bile duct. In the present study, we compared HM47/ A9 antibody expression patterns in HCC and ICC.

\section{Materials and methods}

Cases selected for study. Normal adult livers were obtained from tissue surrounding the liver cancer. Eight-week embryo liver was acquired from a case of ruptured tubal pregnancy and the 20-week embryo liver sample was obtained from a perinatal mortality. We reviewed primary liver cancer cases between January 2002 and December 2012 in the People's Liberation Army 152 Hospital (Henan, China), including 82 cases of HCC, 31 cases of ICC and 11 cases of combined HCC and cholangiocarcinoma (cHCC-CC), which were subjected to resection or puncture. All specimens were fixed in $10 \%$ neutral-buffered formalin, dehydrated in graded alcohol solutions, embedded in paraffin and cut into 4- $\mu$ m-thick sections for hematoxylin and eosin staining, followed by visualization using light microscopy.

Immunohistochemical analysis. Immunohistochemical staining was performed on formalin-fixed, paraffin-embedded tissue sections using the EnVision method. The primary antibodies employed included CD79 $\alpha$ (HM47/A9), AFP (ZSA06), CK19 (A53-B/A2.26), MOC31 (MOC31), CA19-9 (TA888), CEA (Col-1) and Hepatocyte (OCH1E5). All antibodies were purchased from Maxin-Bio Co. (Fuzhou, China). Slides were 

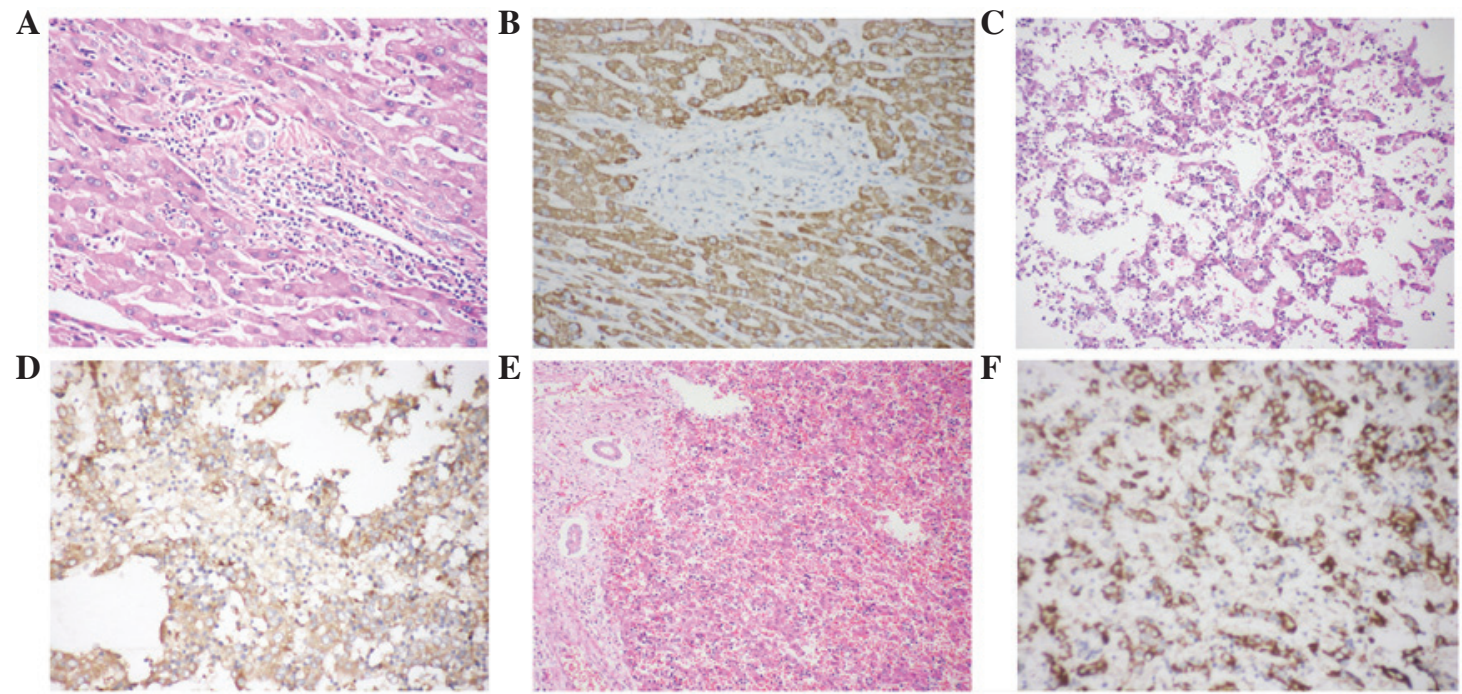

Figure 1. (A) Normal portal area. (B) Normal hepatocytes (99\%) displaying cytoplasmic granular staining positive for HM47/A9. B cells in the portal area show membrane positivity for HM47/A9, while the bile canaliculus and interlobular bile duct are negative for HM47/A9. (C) Eight-week embryo liver. (D) Eightweek embryo hepatocytes (10\%) exhibit granular positivity for HM47/A9. (E) 20-week embryo liver. (F) 20-week embryo hepatocytes (80\%) show granular staining positive for HM47/A9. (A,B,D,F; magnification, x120) (C and E; magnification x60).

counterstained with hematoxylin. To observe mallory hyaline bodies and globular hyaline bodies, slides were counterstained with eosin after immunostaining for $\mathrm{CD} 79 \alpha$.

\section{Results}

Clinical features. The $82 \mathrm{HCC}$ patients included 48 males and 34 females with a median age of 46 years (range, 24-70 years). The 31 ICC patients included 16 males and 15 females with a median age of 51 years (range, 37-74 years). The 11 cases of cHCC-CC occurred in 6 males and 5 females with a median age of 48 years (range, 26-69 years).

Histological, pathological and immunochemistry findings. Normal hepatocytes (Fig. 1A) exhibited diffusely granular, cytoplasmic immunoreactivity to HM47/A9 (Fig. 1B). By contrast, no HM47/A9 positivity was observed in the bile canaliculus or interlobular bile duct (Fig. 1B).

In the 8-week embryo liver (Fig. 1C), $20 \%$ of hepatocytes displayed granular positivity for HM47/A9 (Fig. 1D). Hepatocytes of 20-week embryo liver (Fig. 1E) demonstrated diffuse immunoreactivity to HM47/A9 with a granular pattern in the cytoplasm (Fig. 1F), which was retained throughout life.

HCC cells resembled hepatocytes (Fig. 2A). Some tumors had a plate-like pattern, while other HCC cells formed a pseudoglandular pattern. Tumor cells were round or ovalshaped with abundant granular eosinophilic cytoplasm and single, large central nuclei. Pale bodies and fatty changes were evident. HCC cells tested positive for Hepatocyte (79/82, 96.3\%) and AFP $(24 / 82,29.3 \%)$ and negative for the CEA, CK19, CA19-9 and MOC31. All 82 HCC tumor cells exhibited diffuse granular positivity for HM47/A9 (Fig. 2B).

ICC cells were round or oval, and some were pleomorphic (Fig. 2C and D). Nuclei were small or large with more than one small nucleolus, while the cytoplasm was eosinophilic or vacuolated. Tumor cells formed a tubular gland or cord-like pattern, and all cases were negative for HM47/A9 (Fig. 2E). The tumor cells were positive for the CK19 (31/32, 96.9\%) (Fig. 2F), CEA (7/10, 70\%), CA19-9 (25/32, 78.1\%) and MOC31 (26/32, 81.3\%).

Each of the CC parts in cHCC-CC [11/11 (100\%)] did not express HM47/A9. The majority of HCC components in cHCC-CC [10/11 (90.9\%)] exhibited positive staining for HM47/A9. The excluded case was in a 26-year-old female. These tumor cells demonstrated a similar histology to hepatocytes, with abundant eosinophilic or vacuolated cytoplasm and a centronucleus. Prominent nucleoli were observed in the nucleus. Tumor cells grew as entities and invaded the interlobular bile duct wall (Fig. 3A-C). Significant tumor thrombus in the hemal tube was evident (Fig. 3A). Tumor cells were immunoreactive for Hepatocyte (Fig. 3D), CK19 (Fig. 3F), CEA, CA19-9 and MOC31 (Fig. 3G), but negative for AFP and HM47/A9 (Fig. 3H). CEA (Fig. 3E) immunostaining data revealed no bile canaliculus between carcinoma cells.

Pale bodies, mallory hyaline bodies, fatty degeneration and globular hyaline bodies were negative for HM47/A9 (Fig. 4).

\section{Discussion}

$\mathrm{CD} 79 \alpha$, also known as Ig $\alpha$, is encoded by mouse B cell-specific gene 1 (mb-1) (3). The gene has been identified in pre-B and $\mathrm{B}$ cells, as well as thymocytes and peripheral blood T cells $(3,8)$, but is absent in HeLa and kidney cells (3). In addition, no CD79 $\alpha$ antigen has been detected in the liver, brain, colon, muscle or placenta tissue (5). However, we showed that hepatocytes exhibit strong cytoplasmic granular staining for HM47/A9, while the bile canaliculus and interlobular bile duct are negative for HM47/ A9. Our results are inconsistent with previous reports (5), which may be attributed to our usage of the antibody clone. To date, JCM117 and HM-57 have been widely used by researchers to study CD79 $\alpha$ expression (5), compared with 11E3, 11D10, SP18 and HM47/A9, which have seldom been employed. This discrepancy may be due to the different clones of $\mathrm{CD} 79 \alpha$ antibodies.

CD79 $\alpha$ expression precedes immunoglobulin heavy-chain gene rearrangement and CD20 expression, and disappears later 

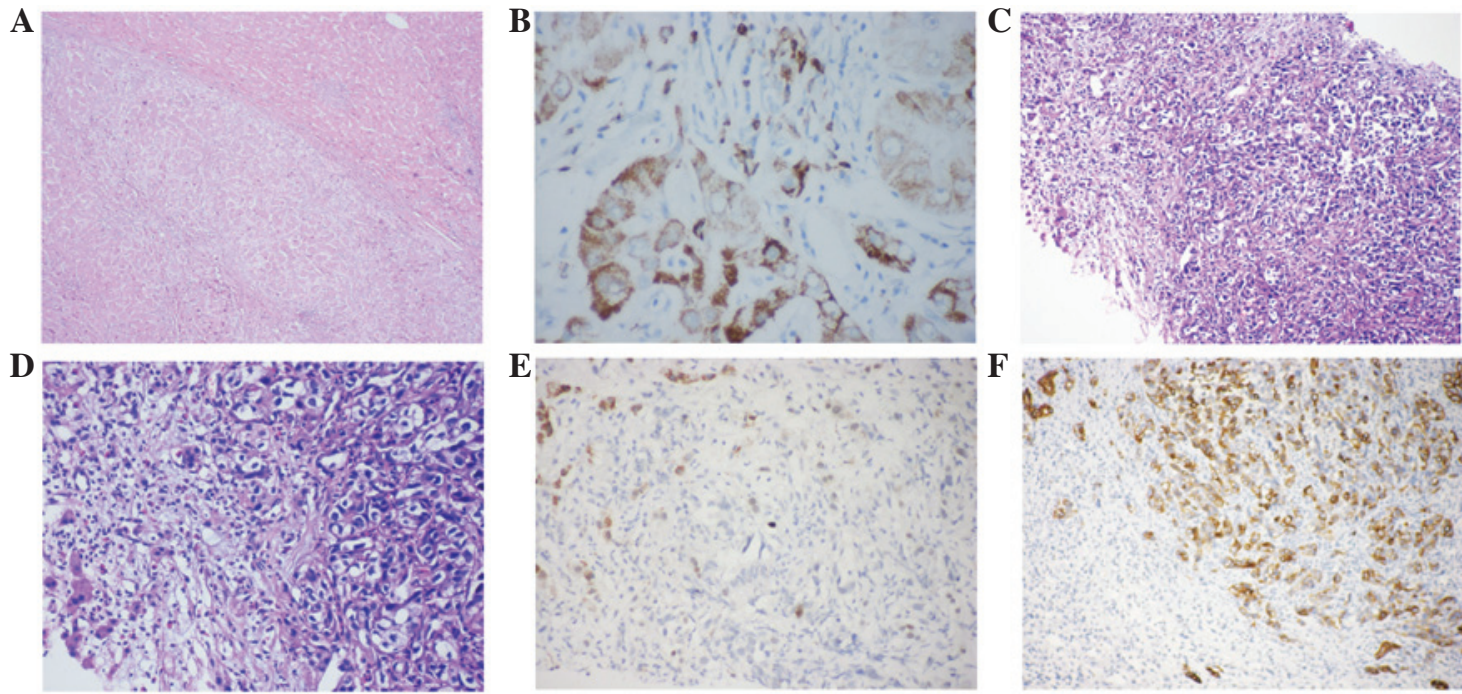

Figure 2. (A) HCC and surrounding liver tissue. Magnification, x24. (B) HCC exhibiting (90\%) positivity for HM47/A9. Magnification, x240 (C and D) Low-power image depicting ICC and residual hepatocytes. Magnification, x60 and x120, respectively. (E) ICC negative for HM47/A9, hepatocytes positive for HM47/A9. Magnification, x120. (F) ICC positive for CK19, hepatocytes negative for CK19. Magnification, x120. HCC, hepatocellular carcinoma; ICC, intrahepatic cholangiocarcinoma.


Figure 3. (A) Tumor thrombus. Magnification, x24. (B and C) cHCC-CC invading the bile duct wall. Tumor cells displaying features of hepatocytes. (D) Tumor cells are immunoreactive for hepatocyte, while bile duct cells are negative for hepatocyte. Magnification, x24 and x120, respectively. (E) Inflammatory cells positive for pCEA, with no bile canaliculus observed between tumor cells. Magnification, x60. (F and G) Tumor cells and bile duct cells positive for MOC31 and CK19, respectively. Magnification, x60 and x120, respesctively. (H) Tumor cells and bile duct cells negative for HM47/A9, B cells positive for HM47/A9 Magnification, x120. cHCC-CC, combined HCC and cholangiocarcinoma.
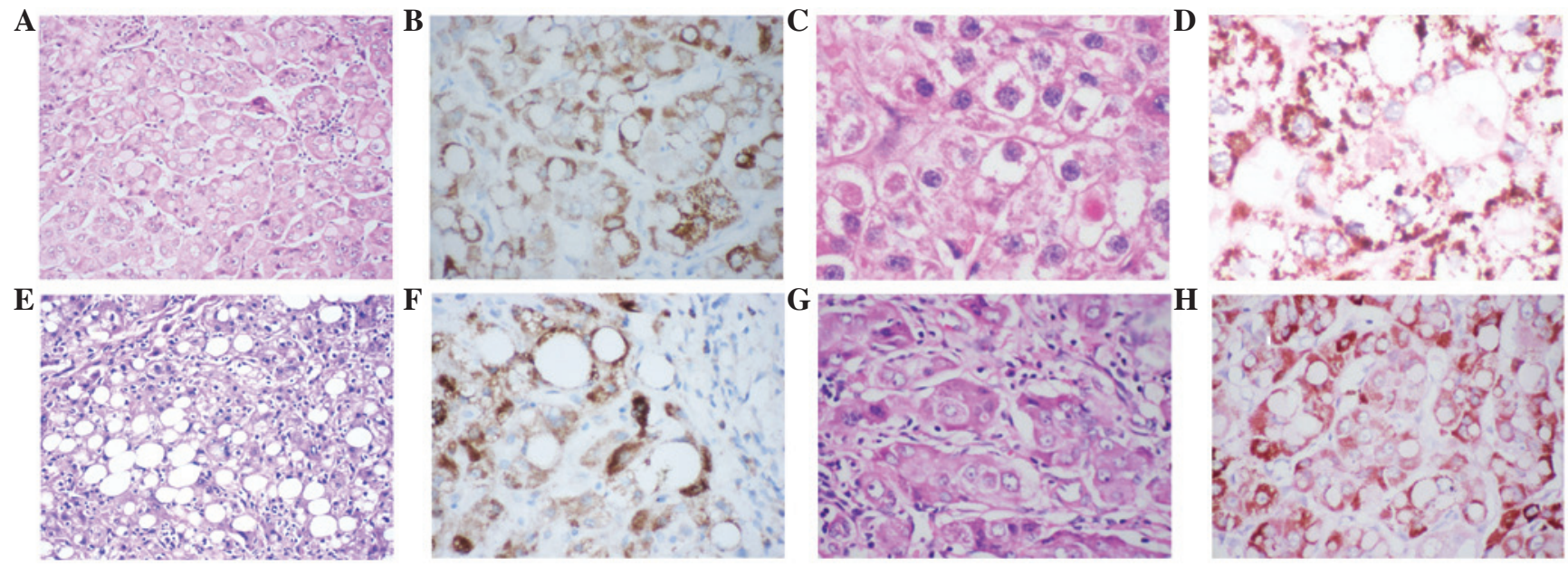

Figure 4. (A) Pale bodies, (C) mallory hyaline bodies, (E) fatty changes, and (G) globular hyaline bodies negative for HM47/A9 (B, D, F and H). (B and F) counterstained with hematoxylin; (D and H) counterstained with hematoxylin and eosin.(A and E; magnification, x120) (B,C,D,F and G; magnification, x240). 
than CD20 in the plasma cell (5). In our experiments, partial $\mathrm{CD} 79 \alpha$ expression in human hepatocytes was observed in the 8 -week embryo, and did not disappear in the adult. Based on these results, we conclude that HM47/A9 expression is activated with human hepatocyte development and maintained constitutively throughout human life. HM47/A9 may also be critically involved in human hepatocyte function. The liver is important in the metabolism of fat, carbohydrate and protein, intermediary metabolism as well as secretion. Further studies are required to elucidate the specific correlation between HM47/A9 and diverse liver functions.

We additionally compared HM47/A9 expression in hepatocytes from normal and diseased livers. As mentioned previously, HM47/A9 was expressed in hepatocytes, but not in the bile canaliculus or interlobular bile duct. In view of these findings, we hypothesized that HM47/A9 expression patterns differ among HCC, ICC and hepatic metastatic carcinoma. In this study, we observed that $82 / 82 \mathrm{HCC}$ cases expressed HM47/A9, while no cases of ICC were positive for HM47/ A9 $(0 / 31)$. These results strongly support the utility of the HM47/A9 antibody in distinguishing HCC from ICC.

None of the $11 \mathrm{CC}$ areas in CHCC-CC expressed HM47/A9. A majority of HCC components (10/11) in cHCC-CC showed positive staining for HM47/A9. The excluded case was a notable case of cHCC-CC, a rare subtype of primary liver carcinoma (9-11). Primary liver cancer cells exhibited features of HCC and invaded the interlobular bile duct. Tumor cells exhibited positivity for Hepatocyte, MOC31, CEA and CK19, and negativity for CD117 and AFP. Based on morphological and immunochemical results, diagnosis should be HCC with bile duct differentiation. Notably, however, these tumor cells were negative for HM47/A9. Earlier research has shown that cHCC-CC displays morphological and clinical similarities with cholangiocarcinoma and leads to poorer prognosis compared with pure HCC (12-14). However, other studies have reported similarities between cHCC-CC and HCC in terms of male/ female ratio, status of hepatitis viral infection and serum AFP level $(9,14)$. These two inconsistent findings may be attributed to different etiological roles according to the geographic situation or the existence of other carcinogenesis mechanisms $(15,16)$. The case in this study displayed normal serum AFP level and no background chronic liver disease, with significant tumor thrombus. Our data support the findings of William RJ, who reported that $\mathrm{cHCC}-\mathrm{CC}$ prognosis is similar to that of cholangiocarcinoma and worse than for pure HCC. Immunochemistry results disclosed tumor cell negativity for HM47/A9. As specified earlier, normal hepatocytes are positive for HM47/A9, while the bile canaliculus and interlobular bile duct are negative for HM47/A9. Thus, the poor prognosis of combined HCC and cholangiocarcinoma may be related to the loss of HM47/A9.

We additionally examined HM47/A9 expression in pale bodies, fatty degeneration, clear cell change, mallory hyaline bodies, intranuclear inclusion and glycogen. Notably, HM47/A9 granular expression was observed around pale bodies, fatty degeneration, clear cell change and mallory bodies, indicating that HM47/A9 is not a component of intermediate filaments, endoplasmic reticulum, glycogen and fat (17-20).

In conclusion, our results strongly suggest that HM47/A9 is an important protein component in hepatocytes that may be effectively employed to differentiate HCC from ICC. HCC with bile duct differentiation had poor prognosis, which may be related to the loss of HM47/A9.

\section{References}

1. Wennerberg AE, Nalesnik MA and Coleman WB: Hepatocyte paraffin 1: a monoclonal antibody that reacts with hepatocytes and can be used for differential diagnosis of hepatic tumors. Am J Pathol 143: 1050-1054, 1993.

2. Porcell AI, De Young BR, Proca DM and Frankel WL: Immunohistochemical analysis of hepatocellular and adenocarcinoma in the liver: MOC31 compares favorably with other putative markers. Mod Pathol 13: 773-778, 2000.

3. Shirakawa H, Kuronuma T, Nishimura Y, Hasebe T, Nakano M, Gotohda N, Takahashi S, Nakagohri T, Konishi M, Kobayashi N, Kinoshita T and Nakatsura T: Glypican-3 is a useful diagnostic marker for a component of hepatocellular carcinoma in human liver cancer. Int J Oncol 34: 649-656, 2009.

4. Herren B and Burrows PD: B cell-restricted human mb-1 gene: expression, function, and lineage infidelity. Immunol Res 26: 35-43, 2002.

5. Torres RM, Flaswinkel H, Reth M and Rajewsky K: Aberrant B cell development and immune response in mice with a compromised BCR complex. Science 272: 1804-1808, 1996.

6. Chu PG and Arber DA: CD79: a review. Appl Immunohistochem Mol Morphol 9: 97-106, 2001.

7. Mason DY, Cordell JL, Brown MH, Borst J, Jones M, Pulford K, Jaffe E, Ralfkiaer E, Dallenbach F, Stein H, Pileri S and Gatter KC: CD79a: a novel marker for B-cell neoplasms in routinely processed tissue samples. Blood 86: 1453-1459, 1995.

8. Bhargava P, Kallakury BV, Ross JS, Azumi N and Bagg A CD79a is heterogeneously expressed in neoplastic and normal myeloid precursors and megakaryocytes in an antibody clonedependent manner. Am J Clin Pathol 128: 306-313, 2007.

9. Yu LM and Chang TW: Human mb-1 gene: complete cDNA sequence and its expression in B cells bearing membrane Ig of various isotypes. J Immunol 148: 633-637, 1992.

10. Ng IO, Shek TW, Nicholls J and Ma LT: Combined hepatocellularcholangiocarcinoma: a clinicopathological study. J Gastroenterol Hepatol 13: 34-40, 1998.

11. Yano Y, Yamamoto J, Kosuge T, Sakamoto Y, Yamasaki S, Shimada K, Ojima H, Sakamoto M, Takayama T and Makuuchi M: Combined hepatocellular and cholangiocarcinoma: a clinicopathologic study of 26 resected cases. Jpn J Clin Oncol 33: 283-287, 2003.

12. Jarnagin WR, Weber S, Tickoo SK, Koea JB, Obiekwe S, Fong Y, DeMatteo RP, Blumgart LH and Klimstra D: Combined hepatocellular and cholangiocarcinoma: demographic, clinical, and prognostic factors. Cancer 94: 2040-2046, 2002.

13. Tickoo SK, Zee SY, Obiekwe S, Xiao H, Koea J, Robiou C Blumgart LH, Jarnagin W, Ladanyi M and Klimstra DS: Combined hepatocellular-cholangiocarcinoma: a histopathologic, immunohistochemical, and in situ hybridization study. Am J Surg Pathol 26: 989-997, 2002.

14. Kim H, Park C, Han KH, Choi J, Kim YB, Kim JK and Park YN: Primary liver carcinoma of intermediate (hepatocyte-cholangiocyte) phenotype. J Hepatol 40: 298-304, 2004.

15. Fujii H, Zhu XG, Matsumoto T, Inagaki M, et al: Genetic classification of combined hepatocellular-cholangiocarcinoma. Hum Pathol 31: 1011-1017, 2000.

16. Park HS, Bae JS, Jang KY, Lee JH, Yu HC, Jung JH, Cho BH, Chung MJ and Moon WS: Clinicopathologic study on combined hepatocellular carcinoma and cholangiocarcinoma: with emphasis on the intermediate cell morphology. J Korean Med Sci 26: 1023-1030, 2011.

17. Nzeako UC, GoodmanZD and Ishak KG: Hepatocellular carcinoma in cirrhotic and noncirrhotic livers. A clinico-histopathologic study of 804 North American patients. Am J Clin Pathol 105: 65-75, 1996.

18. Nakanuma Y and Ohta G: Is mallory body formation a preneoplastic change? A study of 181 cases of liver bearing hepatocellular carcinoma and 82 cases of cirrhosis. Cancer 15: 2400-2404, 1985.

19. Nakashima O, Sugihara S, Eguchi A, Taguchi J, Watanabe J and Kojiro M: Pathomorphologic study of pale bodies in hepatocellular carcinoma. Acta Pathol Jpn 42: 414-418, 1992.

20. Nayar R, Bourtsos E and DeFrias DV: Hyaline globules in renal cell carcinoma and hepatocellular carcinoma. A clue or a diagnostic pitfall on fine-needle aspiration? Am J Clin Pathol 114: 576-582, 2000. 